brucei (14). This protein belongs to a novel class of glycan-anchored membrane proteins (15) that are thought to function in signal transduction (16) and intracellular targeting (17). The presence of this acidic repeat domain suggests that the VHL protein may be localized on the cell membrane and may be involved in signal transduction or cell adhesion. Further clues to the function of the VHL protein may emerge as more sequence information is obtained.

\section{REFERENCES AND NOTES}

1. V. A. McKusick, Mendelian Inheritance in Man (Johns Hopkins Univ. Press, Baltimore, ed. 6, 1983), pp. 534-535; E. R. Maher et al., J. Med. Genet. 28, 443 (1991)

2. K. Tory et al., J. Natl. Cancer Inst. 81, 1097 (1989); E. R. Maher, J. R. Yates, M. A. Ferguson-Smith, J. Med. Genet. 27, 311 (1990).

3. A. Knudson, Proc. Natl. Acad. Sci. U.S.A. 68,820 (1971); Annu. Rev. Genet. 20, 231 (1986).

4. S. Hosoe et al., Genomics 8, 634 (1990); E. R. Maher et al., ibid., p. 957; G. M. Glenn et al., Hum. Genet. 87, 207 (1990); F. Latif et al., Am. J. Hum. Genet. 51 (suppl.). A63 (1992); K. Tory et al. Genomics 13, 275 (1992); F. M. Richards et al., J. Med. Genet. 30, 104 (1993); P. A. Crossey et al., Hum. Mol. Genet., in press; B. R. Seizinger et al., Nature 332, 268 (1988); B. R. Seizinger et al. Proc. Natl. Acad. Sci. U.S.A. 88, 2864 (1991); J. M. Vance et al., Am. J. Hum. Genet. 51 (suppl.), 203 (1992).

5. F. Latif et al., Cancer Res. 53, 861 (1993)

6. M. Yao et al., Hum. Genet., in press.

7. R. White, Curr Opin. Genet. Dev. 2, 53 (1992); R. A. Weinberg, Science 254, 1138 (1991); R. M. Cawthon et al., Cell 62, 193 (1990); M. R. Wallace et al., Science 249, 181 (1990); J. Groden et al., Cell 66, 589 (1991); K. W. Kinzler et al., Science 253, 661 (1991); I. Nishisho et al., ibid., p. 665; J. A. Trafatter et al., Cell 72, 791 (1993)

8. Polyadenylated RNA from all tissue types tested (heart, skeletal muscle, brain, placenta, lung, liver, kidney, pancreas, prostate) showed a strong hybridization signal at 6 to $6.5 \mathrm{~kb}$. In addition, expression was observed by RT-PCR analysis of total RNA from VHL lymphoblastoid cell lines. RNA and corresponding CDNA were prepared and amplified (5) with primers representing the g7 cDNA sequence (Fig. 4)

9. FISH was performed as in K. Tory et al. [Genomics 13, 275 (1992)]. Phage p191, containing an 80-kb insert, was isolated by D. A. Smoller from a three-hit $p 1$ phage genomic library (Genome Systems, Inc., St. Louis, MO).

10. The Southern blots contained DNA samples (purchased from BIOS Laboratories, New Haven, CT) from the following species: human, chimpanzee macaque, cow, rat, mouse, chicken, frog, fruit fly, sea urchin, and yeast (Saccharomyces cerevisiae). Blots were (pre)hybridized in Church buffer [G. M. Church and W. Gilbert, Proc. Natl. Acad. Sci. U.S.A. 81, 1991 (1984)] at $65^{\circ} \mathrm{C}$ for 18 hours and washed in $0.1 \times$ Church buffer at $60^{\circ} \mathrm{C}$ for 60 $\min$.

11. R. F. Doolittle, Protein Sci. 1, 191 (1992)

12. J. W. Fickett, Nucleic Acids Res. 10, 5303 (1982)

13. We searched the databases in GenBank (release 75) with the FASTA family of programs (W. Pearson and D. Lipman, Proc. Natl. Acad. Sci. U.S.A 85, 244 (1988)].

14. M. R. Mowatt, G. S. Wisdom, C. E. Clayton, Mol Cell. Biol. 9, 1332 (1989)

15. M. A. J. Ferguson, Curr. Opin. Struct. Biol. 1, 522 (1991).

16. S. Davis et al., Science 253, 59 (1991).

17. M. P. Lisanti and E. Rodrigez-Boulau, Trends Biochem. Sci. 15, 113 (1990)

18. Previously we did not observe crossovers between the D3S601 locus and VHL in > 300 infor- mative meioses (4). However, we recently detected one such crossover, which allowed us to place the VHL locus distal to D3S601 (E. R. Maher et al. unpublished results).

19. M. Orita et al., Genomics 5, 874 (1989); M. Dean et al., Cell 61, 863 (1990)

20. This work is the result of a 6-year effort and represents a truly communal enterprise. We appreciate the cooperation of the VHL families in the United States, Canada, and Europe. B. Zbar, M. Lerman, and the $\mathrm{NCl}-\mathrm{FCRDC}$ group are grateful to $A$. Rabson for his unwavering support. We thank M. F. Singer for the $\lambda g t 11$ teratocarcinoma cDNA library, H. B. Grossman for RCC cell lines UMRC5 and UMRC6, E. Brundage for help in screening YAC libraries, and J. Klabansky and D. Shores for managing the manuscript. Supported by grants from Action Research and the Cancer Research Campaign (to E.R.M.), the Japanese Overseas Cancer Fellowship of the Foundation for Promotion of Cancer Research (to M.Y.), and the William K. Warren Foundation (to D.S.R.D.).

\title{
Repression of MHC Class I Gene Promoter Activity by Two-Exon Tat of HIV
}

\author{
T. Kevin Howcroft, Klaus Strebel, Malcolm A. Martin, \\ Dinah S. Singer*
}

Major histocompatibility complex (MHC) class I molecules are the major receptors for viral peptides and serve as targets for specific cytotoxic $T$ lymphocytes. Human immuN nodeficiency virus-type 1 (HIV-1) specifically decreased activity of an MHC class I gene promoter up to 12-fold. Repression was effected by the HIV-1 Tat protein derived from a spliced viral transcript (two-exon Tat). These studies define an activity for two-exore Tat distinct from that of one-exon Tat and suggest a mechanism whereby HIV-1-infected cells might be able to avoid immune surveillance, allowing the virus to persist in the infected host.

Major histocompatibility complex molecules play a pivotal role in the initiation and propagation of immune responses. Immune surveillance for viral infections is provided primarily by MHC class I antigens, which bind intracellularly generated viral peptides and act as targets for antiviral cellular immune responses (1). Many viruses are known to repress MHC class I expression, among them a number of retroviruses such as the Moloney leukemia virus (2). Decreases in MHC class I levels provide a mechanism for the virus to evade the host immune response. HIV-1 is a complex retrovirus that primarily infects $\mathrm{CD}^{+}{ }^{+} \mathrm{T}$ cells and monocytes, ultimately causing a depletion in the $\mathrm{CD} 4^{+} \mathrm{T}$ cell population and a profound immunodeficiency (3). Because HIV-1 is known to establish persistent infections, suggesting a viral mechanism to avoid immune surveillance, we have examined its effect on MHC class I expression.

To assess the effect of HIV-1 on MHC class I expression, we transiently transfected human HeLa cells with an HIV $1_{\text {LAI }}$-derived construct, pNL-Al, which expresses all viral gene products except Gag and Pol (4), and measured cell surface

T. K. Howcroft and D. S. Singer, Experimental Immunology Branch, National Cancer Institute, Building 10 , Room 4B-17, NIH, Bethesda, MD 20892.

K. Strebel and M. A. Martin, Laboratory of Molecular Microbiology, National Institute of Allergy and Infectious Diseases, NIH, Bethesda, MD 20892.

*To whom correspondence should be addressed. class I expression by staining with and antibody to human leukocyte antigen W6/32, 48 hours after transfection. Tou distinguish pNL-Al-transfected from non transfected HeLa cells, we also staineed cells with an antibody to gp120 (pNL-A ? encodes gp120). Two-color flow cytometris was performed, and the mean cell surface level of class I on gp $120^{+}$cells was com气 pared with that on gp120- cells in the same population (5). Whereas gp120 g cells stained with a mean fluorescence intensity (MFI) of $100 \pm 8$, gp $120^{+}$cell: expressed significantly lower levels of MHC class I, with an MFI of $49.2 \pm 2$. $(P<0.0004$, five independent experf ments). Thus, one or more HIV-1 gene products (but not Gag or Pol) are capable of markedly decreasing endogenous MHC class I expression. The extent of reduction observed in the present studies reflects only the difference that occurs during the transient 48-hour assay and could be an underestimate of the actual reduction of class I expression occurring in transfected HeLa cells. These results are consistent with a previous report describing a transient decrease in class I expression in HIV-1-infected T cell lines (6). Changes in class I expression of the magnitude observed here have been shown to alter susceptibility of cells to cytolysis by cytotoxic T cells (7).

To determine whether the HIV-1-mediated reduction of class I expression was transcriptional, we examined the effect of 
HIV-1 gene products on an MHC class I promoter ligated to the reporter gene chloramphenicol acetyltransferase (CAT). A class I promoter construct containing 313 base pairs (bp) of $5^{\prime}$ flanking sequences (8) was cotransfected into HeLa cells with varying amounts of either pNLA1 or a control construct, pAR, which expresses only a subset of the pNL-A1encoded viral proteins (Tat, $\mathrm{Vpu}$, an aberrant Env/Nef fusion protein, and a truncated Gag protein) (4). Cotransfection of the class I promoter with pNL-A1 markedly reduced promoter-directed CAT activity to $11.7 \pm 1.5 \%$ of controls cotransfected with carrier DNA alone. In contrast, cotransfection with the pAR construct did not reduce class I promoter activity (9).

The repression mediated by pNL-A1 was not due to nonspecific effects of HIV-1encoded products on the class I promoter, because expression of CAT under the control of SV40, Rous sarcoma virus (RSV), or murine leukemia virus (MuLV) promoters was not significantly affected when the promoter was cotransfected with pNL-A1 into HeLa cells (Table 1). Because the constructs examined were identical except for the upstream promoter region, the failure of pNL-A1 to decrease CAT activity other than in the class I promoter construct also eliminates possible posttranscriptional effects. Furthermore, pNL-A1 inhibited the expression of a second reporter gene, luciferase, directed by the class I promoter [(10); see also Table 2], indicating that the pNL-A1 effect did not specifically target the CAT reporter. These experiments demonstrate that HIV-1-mediated repression of MHC class I expression occurs transcriptionally.

To map the target site of the HIV-1mediated repression, we tested deletion constructs of the class I promoter for their sensitivity to pNL-A1. A variety of regulatory elements have been identified between -209 and $-68 \mathrm{bp}$ in the $5^{\prime}$ flanking region of the MHC class I gene (11), including a constitutive enhancer (enhancer A), an interferon response element, a cAMP (adenosine $3^{\prime}, 5^{\prime}$-monophosphate) response element (CRE), and a weak enhancer (enhancer B). Enhancer $A$ has been shown previously to bind a homodimer of the $\mathrm{p} 50 \mathrm{NF \kappa B}$; alternative processing of the NFKB precursor after HIV-1 infection has been reported (12). Deletion to within $68 \mathrm{bp}$ of the initiation of transcription did not abrogate repression by pNL-A1 (Fig. 1). Thus, the effect was not mediated by enhancer A-NFkB, the CRE, or the enhancer $B$ regions, all of which are deleted from this construct. Rather, the HIV-1-responsive element maps to within the 68 -bp region of the minimal promoter, a region that is only known to contain the CAT and TATA boxes.

Because pNL-A1 but not $\mathrm{pAR}$ reduced class I promoter activity, HIV-1 sequences responsible for class I promoter repression map to a region of the proviral genome $3^{\prime}$ of the pol gene (Fig. 2). Contained within this region are seven genes: vif, vpu, vpr, rev, tat, env, and nef. Mutations affecting six of these genes were introduced into the pNL-A1 construct and cotransfected into HeLa cells with the class I promoter constructs, -135CAT and -313CAT. Each of these mutant derivatives of pNL-A1 efficiently repressed the class I promoter (Fig. 2) $(10,13)$. Indeed, deletion of nef significantly augmented the repression of the class I promoter. Thus, the six HIV-1 gene products-Nef, Rev, Vif, $\mathrm{Vpr}, \mathrm{Vpu}$, or Env-were not responsible for repressing the promoter. The effects of the Nef and Rev proteins were also tested directly; expression vectors containing rev or nef under the control of a cytomegalovirus (CMV) promoter did not reduce class I promoter activity over a range of concentrations ( 1 to $6 \mu \mathrm{g}$ of plasmid DNA) tested (10). Of the seven gene products encoded by pNL-A 1 , the only one not ruled out in this experiment is Tat (14).

Tat is a viral-encoded regulatory protein that transactivates HIV long terminal repeat (LTR)-directed gene expression. Tat is synthesized as either an 86 (or somewhat longer)-amino acid protein de-

Table 1. pNLA1 selectively reduces class I promoter activity. HeLa cells were cotransfected (9) with the indicated promoter CAT construct $(5 \mu \mathrm{g})$ and with the HIV-1 proviral constructs pAR or pNL-A1 $(5 \mu \mathrm{g})$. Values (an average of four duplicate samples) represent CAT activity normalized to control transfections without an HIV-1 proviral construct. All assays were normalized to $\beta$-galactosidase activity.

\begin{tabular}{lll}
\hline Promoter & pAR/control & pNL-A1/control \\
\hline Class I & $1.35 \pm 0.095$ & $0.46 \pm 0.02$ \\
SV40 & $1.29 \pm 0.13$ & $1.51 \pm 0.26$ \\
RSV & $0.99 \pm 0.003$ & $1.00 \pm 0.003$ \\
MuLV & $3.16 \pm 0.04$ & $1.90 \pm 0.135$ \\
\hline
\end{tabular}

rived from a spliced mRNA (two-exon Tat) or a 72-amino acid product containing only the first coding exon (one-exon Tat), the latter appearing after the onset of Rev expression. Whereas pNL-A1 directs the synthesis of both forms of Tat, only the single-exon form is expressed by pAR. Although both Tat species transactivate the HIV LTR in tissue culture systems (15), the functional significance of the two forms of Tat in vivo is not presently understood. Because pNL-A1 but not pAR inhibited the class I promoter, the results obtained in the pNL-A1 mutagenesis experiment (Fig. 2) suggested that the observed repression was due to the two-exon species of Tat.

To examine this possibility further, we cotransfected expression vectors encoding HIV- $1_{\text {LAI }}$-derived two-exon Tat (86 amino acids in length) and one-exon Tat (72 amino acids in length) into HeLa cells with a class I promoter construct $(-135 \mathrm{Lu}-$ ciferase) (16). Two-exon Tat, in the absence of any other HIV-1 transcripts, markedly decreased class I promoter activity, whereas one-exon Tat had only a minor effect (Table 2). This suggests that the

Table 2. Repression of class I promoter activity by two-exon Tat in the absence of any other viral gene products. HeLa cells were cotransfected (9) with a class I promoter construct containing $135 \mathrm{bp}$ of promoter proximal sequence ligated to the luciferase reporter gene $(-135$ luciferase; $5 \mu \mathrm{g})$ and the indicated Tat expression vectors $(5 \mu \mathrm{g})$ or with a control expression vector consisting of the SV40 early promoter with a nonfunctional insert (16). The effect of Tat on MHC class I promoter activity is expressed relative to that of the control expression vector. Luciferase activity was determined on a Monolight 2010 luminometer (Analytical Luminescence Laboratory) and was normalized to $\beta$-galactosidase activity.

\begin{tabular}{|c|c|c|c|}
\hline Tat & Form & Origin & $\begin{array}{c}\text { Relative } \\
\text { promoter activity } \\
\text { (Tat/control) }\end{array}$ \\
\hline $\begin{array}{l}\text { Tat } 86 \\
\text { Tat } 72 \\
\text { Tat } 49 \\
\text { Tat } 101\end{array}$ & $\begin{array}{l}\text { Two-exon } \\
\text { One-exon } \\
\text { Truncation } \\
\text { Two-exon }\end{array}$ & $\begin{array}{l}\text { HIV-1 } \\
\text { HIV-1 LAI } \\
\text { HIV-1 LAI } \\
\text { HIV-1 }\end{array}$ & $\begin{array}{l}0.172 \pm 0.009 \\
0.767 \pm 0.088 \\
0.867 \pm 0.089 \\
0.412 \pm 0.024\end{array}$ \\
\hline
\end{tabular}

Fig. 1. HIV-1-mediated negative regulation of the class I promoter requires only 68 bp of promoterproximal sequences. Truncated MHC class I promoter CAT constructs $(5 \mu \mathrm{g})$ were cotransfected with pAR or pNL-A1 $(5 \mu \mathrm{g})$ into HeLa cells, and CAT activity was determined (9). Results are expressed relative to control values. Figure summarizes data obtained from two independent experiments performed in duplicate.
Belative effect of HIV constructs PAR/control PNL-A1/control

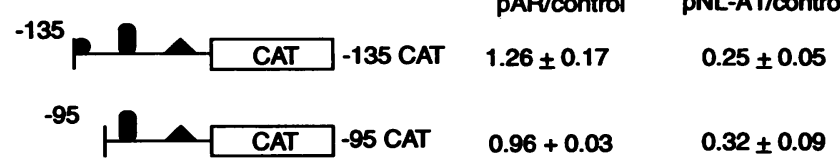

$-68$

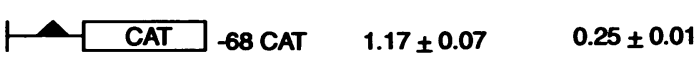



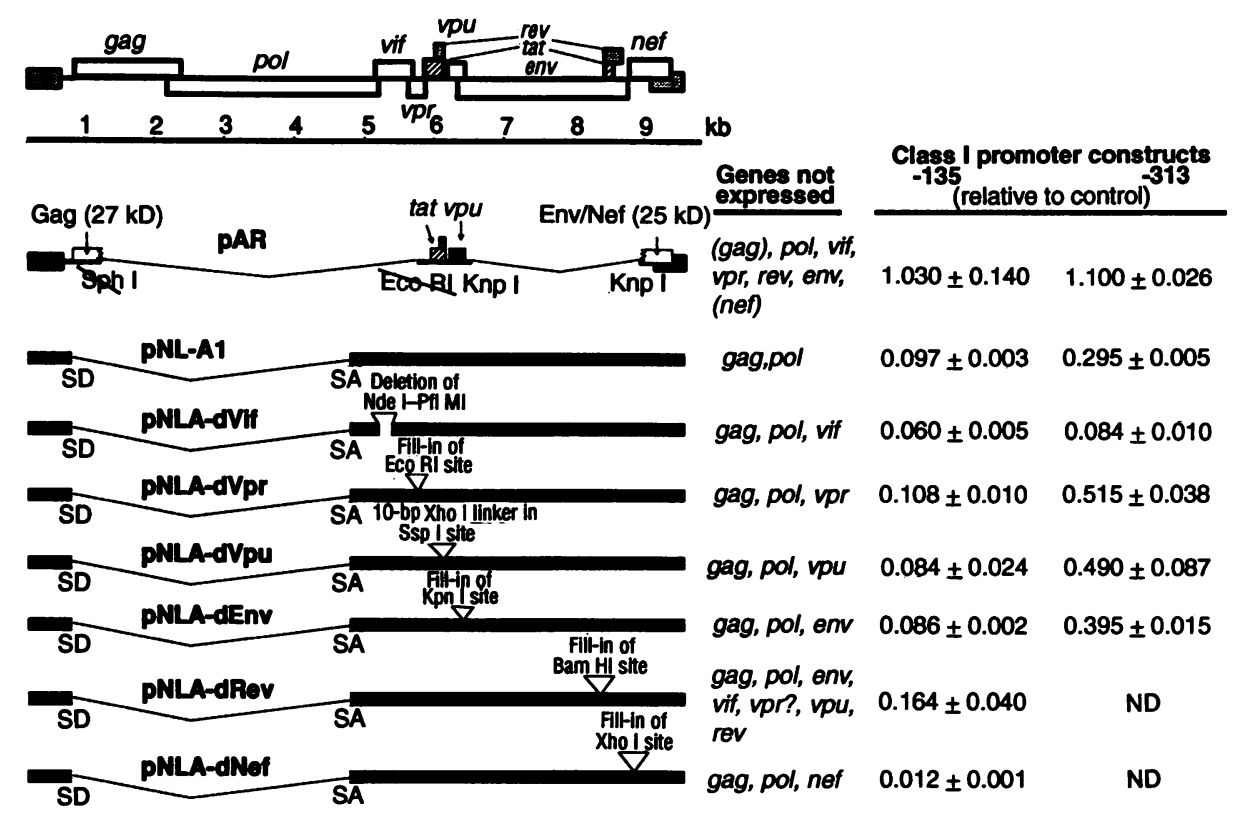

Fig. 2. Repression of class I promoter activity not eliminated by HIV-1 mutations that eliminate the expression of Gag, Pol, Vif, Vpr, Vpu, Rev, Nef, or Env. Derivative pNL-A1 constructs $(5 \mu \mathrm{g})$ containing the indicated mutations to eliminate expression of specific HIV-1 genes were generated and cotransfected along with -135 CAT or -313 CAT $(5 \mu \mathrm{g})$ into HeLa cells, and CAT activity was determined (9). Results are expressed relative to control values.

repression of the class I promoter by pNLA1 but not by its derivative pAR resulted from its capacity to express two-exon Tat and indicates that the $\mathrm{COOH}$-terminal domain of two-exon Tat is essential for repression. These experiments define a function of two-exon HIV-1 Tat distinct from that of one-exon Tat and indicate that the HIV-1 Tat protein can act both as a transcriptional repressor as well as a transcriptional activator.

A two-exon Tat (101 amino acids in length) derived from the HIV-1 ${ }_{\mathrm{SF} 2}$ isolate also repressed class I promoter activity, but to a lesser extent than the HIV-1 $\mathrm{LAI}^{-}$ derived two-exon Tat (Table 2). This may reflect differences in the amino acid sequence between positions 73 and 86 or in the 15 additional residues in the HIV- $1_{\text {SF2 }}$ Tat. The present studies raise the possibility that differences in the strength of Tat-mediated repression of class I expression may influence the persistence and pathogenicity associated with different viral strains.

The mechanism by which two-exon Tat represses class I promoter activity is not known. Tat may act directly by regulating class I promoter activity or function indirectly by transactivating a gene that regulates class I promoter activity. Tat has been reported to interact with cellular factors such as Tat-binding protein (TBP) -1 and mammalian suppressor of sgv1 (MSS1) during the transactivation of the HIV LTR (17). Whether these factors are involved in regulating class I gene expression, either alone or in concert with Tat, is not known.

Because MHC class I molecules are the primary presenters of viral peptides to $T$ cells, reduction in cell surface levels of class I might lead to a reduced ability of an infected cell to be recognized by HIV-1specific cytotoxic T lymphocytes. Downregulation of class I molecules in an HIV1 -infected cell could provide a mechanism for that cell to avoid normal immune surveillance.

\section{REFERENCES AND NOTES}

1. A. Townsend and H. Bodmer, Annu. Rev. Immunol. 7, 601 (1989); R. Demars and T. Spies, Trends Cell Biol. 2, 81 (1992)

2. L. R. Goding and W. S. M. Wold, CRC Crit. Rev. Immunol. 10, 53 (1990); D. J. Maudsley and J. D. Pound, Immunol. Today 12, 429 (1991).

3. B. R. Cullen, Annu. Rev. Microbiol. 45, 219 (1991); W. A. Haseltine, FASEB J. 5, 2349 (1991); Y. N Vaisknov et al., Annu. Rev. Biochem. 60, 577 (1991).

4. K. Strebel, T. Klimkatt, M. A. Martin, Science 241, 1221 (1988).

5. HeLa cells were transfected (9) with $30 \mu \mathrm{g}$ of pNL-A1 or a control plasmid containing the viral LTR (pBennCAT). After 48 hours, cells were harvested and stained with fluorescein isothiocyanate-conjugated monoclonal antibody to human MHC (W6/32) (provided by J. Titus, NIH) and a biotin-conjugated antibody to gp120 (American Biotechnologies), which was developed with phosphotidlylethanolamine-avidin. Cells $(50,000)$ were analyzed by flow cytometry on a Becton Dickinson FACScan. Background mean fluorescence was 19.4. The gp120+ population among the cells transfected with pNL-A1 was defined as those cells with a fluorescence intensity above that of cells transfected with the control plasmid and averaged $-3 \%$ of the cells. Control transfections using a plasmid containing an HIV-1 LTR ligated to the CAT gene did not show any gp $120^{+}$-staining cells nor any reduction in class 1 expression. The data represent the results of five independent transfections.

6. J. A. Scheppler et al., J. Immunol. 143, 2858 (1989).

7. M. Bevan, Immunol. Today 5, 128 (1984); R. Flores and P. Gilmer, J. Immunol. 132, 2767 (1984).

8. The MHC class I promoter used in these studies was derived from the swine class I gene PD1 [R. Ehrlich, J. Maguire, D. Singer, Mol. Cell. Biol. 8 695 (1988)]. The various deletion constructs were generated by polymerase chain amplification and were ligated into the Bam HI-Hind III site of the pSV3CAT reporter gene. Deletion constructs were sequenced to determine authenticity

9. HeLa cells $\left(1 \times 10^{6}\right.$ cells in Dulbecco's minimum essential medium supplemented with $2 \mathrm{mM}$ L-glutamine, Hepes, gentamicin sulfate, and $10 \%$ fetal bovine serum seeded 24 hours before transfection in a $100-\mathrm{mm}^{2}$ tissue culture dish) were cotransfected by standard calcium phosphate precipitation (8) with $10 \mu \mathrm{g}$ of $-313 \mathrm{CAT}, 100 \mathrm{ng}$ of CMV- $\beta$-galactosidase (as an internal control), and $10 \mu \mathrm{g}$ of the HIV-1 proviral constructs, pAR or pNL-A1. (In other experiments, where less test DNA was used, carrier salmon sperm or pUC 19 DNA was added to each sample to achieve a constant total amount of $20 \mu \mathrm{g}$ of transfected DNA.) Cells were harvested after 48 hours, and extracts were prepared. CAT assays were performed after normalization to $\beta$-galactosidase. Data were analyzed with an AMBIS 4000 radioanalytic imaging detector. Results are expressed relative to the control and summarize data obtained from six independent transfections, each of which was performed in triplicate.

10. T. K. Howcroft and D. S. Singer, unpublished data.

11. P. A. Burke et al., J. Exp. Med. 169, 1309 (1989): M. A. Blanar et al., EMBO J. 8, 1139 (1989); M. Kiernan et al., Cell 62, 1007 (1990); D. S. Singer and J. E. Maguire, CRC Crit. Rev. Immunol. 10, $235(1990)$

12. Y. Rivière, V. Blank, P. Kourilsky, A. Israël, Nature 350, 625 (1991).

13. The -135CAT construct is generally more efficiently repressed by pNL-A1 than is -313CAT. The molecular basis for this is not currently understood. The possibility that DNA sequence elements located between -135 and -313 bp may modulate repression is being investigated.

14. Other possibilities have been eliminated as follows. (i) Inhibition of class I promoter activity might occur as a result of direct competition for transcription factors by HIV-1 proviral DNA sequences. This possibility is unlikely because neither the HIV-1 LTR alone nor PNL-A1 DNA sequences lacking the HIV-1 LTR repress class I promoter activity (10). (ii) Because pAR does not produce either infectious virus or viral particles, simple sequestration of transcription factors within a viral particle cannot account for the lack of repression.

15. J. Sodroski et al., Science 229, 74 (1985); B. R. Cullen, Cell 46, 973 (1986); M. H. Malim, J. Hauber, R. Fenrick, B. R. Cullen, Nature 335, 181 (1988).

16. The expression vectors encoding Tat72, Tat101, and Tat49 use the SV40 early promoter and were provided by $\mathrm{K}$. T. Jeang (NIH); Tat49 was derived by truncation after amino acid 49 of the Tat72 construct. The Tat86 vector uses the SV40 late promoter and was provided by J. Brady (NIH).

17. TBP-1 has been demonstrated to decrease, whereas MSS1 increases, Tat promoter activity in such systems [P. Nelbock, P. J. Dillon, A. Perkins, C. A. Rosen, Science 248, 1650 (1990); H. Shibuya et al., Nature 357, 700 (1992)].

18. We thank J. Brown and J. Weissman for helpful discussions, D. Nikodem for technical assistance, and G. Shearer, S. Berger, and A. Singer for critical review of the manuscript.

15 January 1993; accepted 22 March 1993 